\title{
Multimodal, Biomaterial-Focused Anticoagulation via Superlow Fouling Zwitterionic Functional Groups Coupled with Anti-Platelet Nitric Oxide Release
}

Kagya Amoako

University of New Haven, kamoako@newhaven.edu

Harihara S. Sundaram

University of Washington

Ahmed Suhaib

The University Of Michigan

Shaoyi Jiang

University of Washington

Keith E. Cook

Carnegie Melon University

Follow this and additional works at: http://digitalcommons.newhaven.edu/mechanicalengineeringfacpubs

Part of the Biomedical Engineering and Bioengineering Commons, and the Mechanical Engineering Commons

\section{Publisher Citation}

Amoako, K. A., Sundaram, H. S., Suhaib, A., Jiang, S. and Cook, K. E. (2016), Multimodal, Biomaterial-Focused Anticoagulation via Superlow Fouling Zwitterionic Functional Groups Coupled with Anti-Platelet Nitric Oxide Release. Advanced Materials Interfaces. Article first published online: 19 JAN 2016 doi: 10.1002/admi.201500646

\section{Comments}

This is the peer reviewed version of the following article: Amoako, K. A., Sundaram, H. S., Suhaib, A., Jiang, S. and Cook, K. E. (2016), Multimodal, Biomaterial-Focused Anticoagulation via Superlow Fouling Zwitterionic Functional Groups Coupled with Anti-Platelet Nitric Oxide Release. Advanced Materials Interfaces, which has been published in final form at http://dx.doi.org/10.1002/admi.201500646. This article may be used for non-commercial purposes in accordance with Wiley Terms and Conditions for Self-Archiving. 


\section{WILEY-VCH}

Multimodal, Biomaterial-Focused Anticoagulation Via Super-low Fouling Zwitterionic Functional Groups Coupled with Anti-Platelet Nitric Oxide Release

Kagya A Amoako*, Harihara S Sundaram, Ahmed Suhaib, Shaoyi Jiang, and Keith E. Cook*

Dr. Kagya Amoako Author 1,

College of Engineering (Biomedical Engineering) University of New Haven, West Haven, CT 06516, USA

Kamoako@newhaven.edu

Dr. Harihara S Sundaram Author 2, Dr. Shaoyi Jiang Author 3

Department of Chemical Engineering, Seattle WA 21227, USA

Ahmed Suhaib Author 4

Department of Biomedical Engineering, University of Michigan, Ann Arbor USA

Dr. Keith Cook Author 5

Department of Biomedical Engineering, Carnegie Mellon University, 700 Technology Drive, Pittsburgh, PA 15219, USA

keicook@andrew.cmu.edu

Keywords: zwitterionic polycarboxybetaine, anti-fouling coatings, nitric oxide, platelet adsorption, blood-contacting surfaces 


\section{WILEY-VCH}

Abstract:

The functions of anti-fouling, zwitterionic polycarboxybetaine $(\mathrm{pCB})$ and anti-platelet nitric oxide (NO) release replicate key anticoagulant properties of the endothelium. The two approaches, only tested separately thus far, were paired on gas permeable polydimethylsiloxane (PDMS) membranes and evaluated for anti-coagulation. Uncoated PDMS (control) and PDMS coated with pCB were screened for fibrinogen (Fg) fouling followed by platelet adsorption testing to evaluate the effects of coating and/or NO using bioreactors. Bare or coated PDMS membranes separated sheep plasma $\left(10^{8}\right.$ platelets $\left./ \mathrm{ml}\right)$ and gas flow chambers within the bioreactors. Either 100 or $0 \mathrm{ppm}$ of $\mathrm{NO} / \mathrm{N}_{2}$ flowed through the gas chamber for NO release at the plasma/biomaterial interface. Surface-adsorbed platelets were quantified using a lactate dehydrogenase assay after 8 hrs of plasma recirculation. Fg fouling and platelet adsorption on pCB-coated PDMS were $10.40 \pm 3.0 \%$ of control $(\mathrm{p}<0.01)$ and $23.3 \pm 7.4 \%(p<0.01)$ of control respectively. NO flux alone limited platelet adsorption to $79.0 \pm 5.0 \%(\mathrm{p}<0.05)$ of control. Together, $\mathrm{NO}$ and $\mathrm{pCB}$ reduced platelet adsorption to $6.90 \pm$ $1.30 \%$ of the uncoated control $(\mathrm{p}<0.001)$. The data suggest that $\mathrm{pCB}$ coating and NO act in concert to reduce platelet fouling at significantly higher levels than either pCB coating or NO release alone.

\section{Introduction}

Blood contacting medical devices typically fail in the long-term due to clot formation.

Significant advances have been made towards achieving materials capable of longer-term use, including 1) heparin coatings to reduce thrombin generation $\left.{ }^{[1-3]}, 2\right)$ various coatings that resist adsorption of plasma proteins ${ }^{[4-6]}$, and 3) nitric oxide gas to reduce platelet activation. ${ }^{[7-11]}$ Each technology is inspired by a single mechanism of the rather multimodal approach used by the endothelium to control coagulation. Thus, each method provides a modest decrease in 


\section{WILEY-VCH}

surface clotting, but none has yielded the breakthrough needed to markedly slow device failure due to clot formation.

This paper describes a first step toward achieving an "artificial endothelium" to improve the functional lifetime blood-contacting devices such as artificial organs, vascular grafts, catheters, and cannulas. We couple anti-fouling zwitterionic functional groups, similar to those expressed by endothelial cells ${ }^{[12-13]}$ with anti-platelet nitric oxide (NO), which is released by endothelial cells. ${ }^{[14-16]}$

The rationale for using these two systems is graphically described in Figure 1.

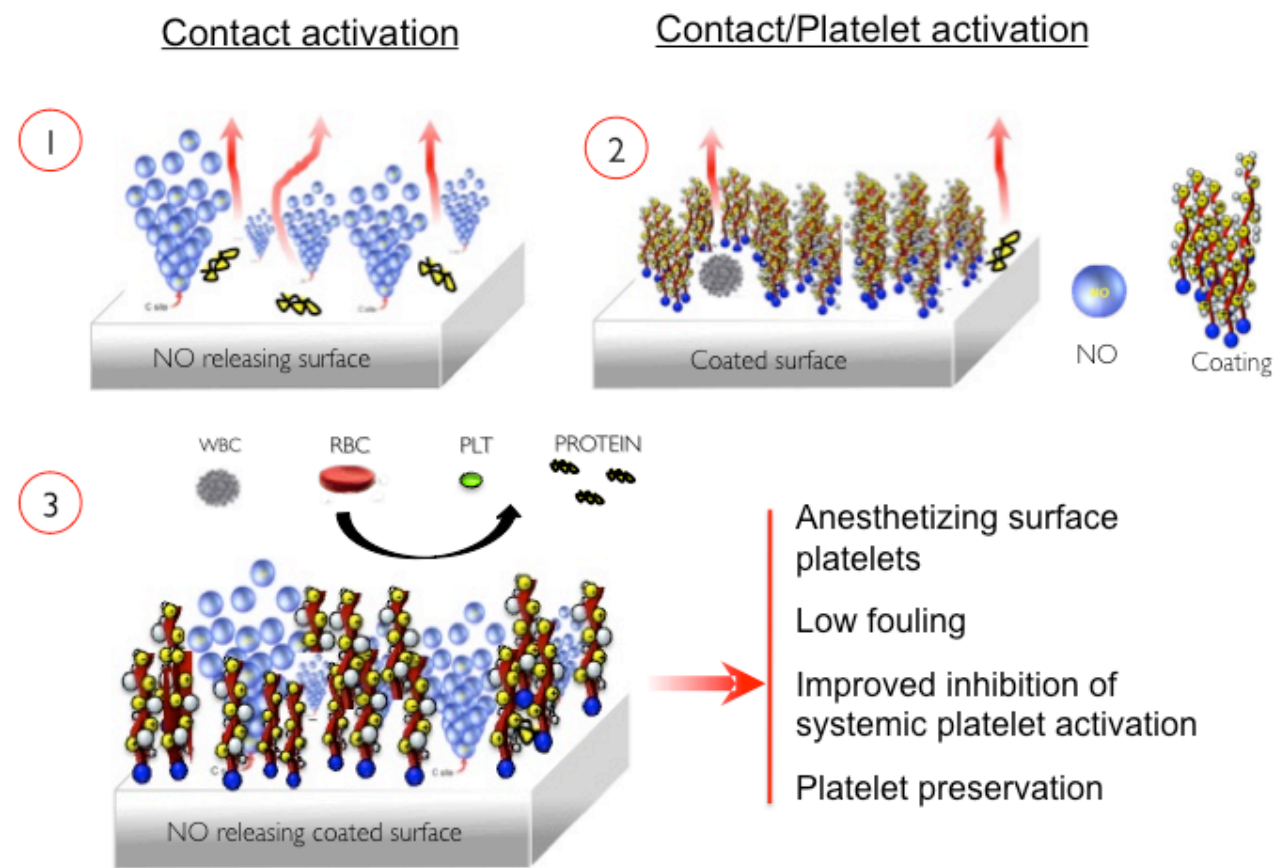

Figure 1. (A bio-inspired hypothesis that the conjugation of surface anti-platelet NOrelease/protein anti-fouling coatings on a surface can better limit blood activation; A surface modified for NO release alone, 1), will have to compete with contact activation of procoagulant proteins, and those modified with protein anti-fouling coatings alone, 2), will require unattainably high graft densities to remain non-fouling. However, when NO release is combined with surface coatings, 3 ), the anti-platelet and low protein fouling conjugate effect can lead to lower blood activation.)

Surfaces modified for NO release alone (1), will not be able to resist protein adsorption, have to compete with procoagulant proteins including the adsorption of fibrinogen, which is shown to increase on NO releasing surfaces. ${ }^{[17]}$ Surfaces modified with protein adsorption resistant coatings alone (2) will require very high, uniform graft densities to remain non-fouling. Any 


\section{WILEY-VCH}

imperfection in the coating can lead to fouling, and trigger coagulation. However, when NO release is combined with surface coatings, 3), procoagulant protein adsorption and subsequent platelet adhesion can be greatly limited by the coating while NO release inhibits platelets that approach regions of coating imperfection.

Prior to this paper, we studied how surface NO flux and PMEA coatings synergistically affect platelet adsorption. ${ }^{[17]}$ It was concluded in that paper that the PMEA significantly inhibits adsorption. However, more than $69.7 \pm 6.8 \%$ reduction in platelet adsorption was achieved only when the PMEA was paired with high levels of NO flux. These findings led us to explore other anti-fouling coatings, which may exhibit a higher anti-fouling property. Published studies have demonstrated a lesser amount of protein fouling with pCB than PMEA coating. ${ }^{[17,18]}$ We thus hypothesized that paring pCB with NO concentrations would lead to less platelet binding, and that this might allow for the use of lower NO surface flux.

The anti-fouling property of polycarboxybetaine coatings has been well studied. On their own, pCB grafted surfaces have shown undetectable nonspecific protein adsorption $\left(<0.3 \mathrm{ng} \mathrm{cm}^{-2}\right)$ from single-protein solutions ${ }^{[19]}$ or complex media ${ }^{[20,21]}$ by surface plasmon resonance. Nitric oxide releasing or generating surfaces are capable of reducing platelet adsorption by approximately $40 \%{ }^{[22,11]}$, and markedly reduce clot formation in artificial lungs. ${ }^{[23]}$ Unfortunately, in applications requiring large surface- area-to-volume-ratios (e.g. artificial lungs and kidneys) or small bore architecture (vascular grafts), NO secretion or zwitterionic grafting alone may not be adequate to counter coagulation and preserve device function, particularly when device use of weeks to months is required. Nitric oxide release alone doesn't limit protein adsorption, and thus, platelets can still bind and activate at the surface. Indeed, the adsorption of procoagulant fibrinogen has been shown to increase on nitric oxiderich surfaces. ${ }^{[24]}$ Anti-fouling coatings allowing even a small amount of protein adsorption will still allow some degree platelets binding that could promote thrombus formation. 


\section{WILEY-VCH}

In the current study, PDMS membranes were either left uncoated or coated with pCB. Coated and uncoated surfaces were also made to release NO. NO flux has been achieved via materials approaches by incorporating a finite amount of NO donors into the substrate ${ }^{[22]}$ or by catalytically generating NO via surface embedded micro and nano $\mathrm{Cu}$ particles. ${ }^{\text {[23] }}$ However, NO delivery in gas exchangers like artificial lungs can be achieved by direct flow of NO gas. ${ }^{[25]}$ To this effect, a bioreactor system was designed so that NO gas diffuses from the uncoated side of membranes to its pCB-coated surface. This system was used to test the synergistic effect of $\mathrm{pCB}$ coating and NO release on platelet adsorption from plasma.

\section{Results}

PDMS and pCB coated PDMS (PDMS-pCB) materials were analyzed using XPS. The survey scan is showed in Figure 2A and 2B. CBMA coating on PDMS is about $4 \mathrm{~nm}$ (based on experiments on CBMA coatings on gold coated glass chips). The surface of PDMS coated with DOPA-pCB showed the presence of nitrogen $(\mathrm{N} \mathrm{1s}$, about 2.4 atomic percent) and the silicon content reduced from 28.8 percent to 17.3 percent. See Table 1.

Table 1. (Atomic percent of surface elements of uncoated PDMS and pCB coated PDMS.)

\begin{tabular}{|l|l|l|l|l|}
\hline & C 1s & O 1s & Si 2p & N 1s \\
\hline PDMS & 45.29 & 25.87 & 28.82 & \\
\hline PDMS-pCB & 54.05 & 24.79 & 17.306 & 2.34 \\
\hline
\end{tabular}

High-resolution nitrogen spectra of uncoated PDMS and DOPA-pCB coated PDMS are shown in Figure 2C and 2D respectively. High-resolution N 1s spectra of PDMS coated with DOPA-pCB (Fig. 4D) exhibited two distinct peaks (N 1s, Pk01 $400.2 \mathrm{eV}$ and Pk02 402.7 $\mathrm{eV})$. The lower binding energy peak was assigned to $\mathrm{N}$ bound to $\mathrm{C}(\mathrm{C}-\mathrm{N}$ of polydopamine and $\mathrm{pCB})$ while the higher binding energy peak was assigned to quaternary nitrogen $\left(\mathrm{R}_{3} \mathrm{~N}^{+}\right.$of pCB). ${ }^{[26]}$ This confirms the presence of polymeric coating on top of PDMS. Clealy PDMS did 


\section{WILEY-VCH}

not show any peaks in the region between $380-400 \mathrm{eV}$ whereas the polymer coated surface showed significant presence of nitrogen (Figure 2C).
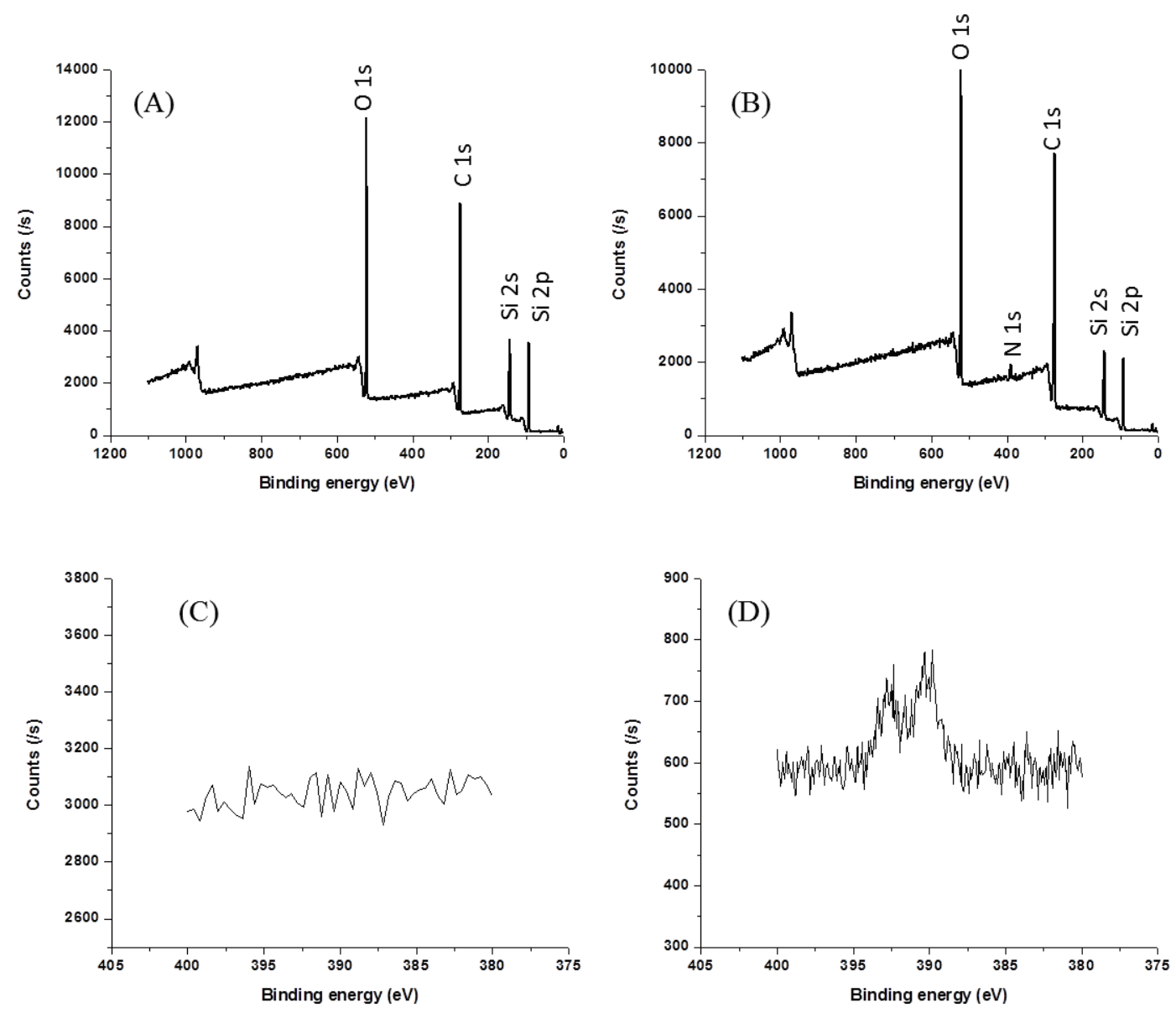

Figure 2. (XPS survey scan of PDMS (A) and PDMS-pCB (B). High resolution nitrogen spectra for PDMS (C) and PDMS-pCB (D).)

PDMS coated with DOPA-pCB showed significant inhibition of fouling compared uncoated controls. The inhibition of fibrinogen adsorption on DOPA-pCB coated PDMS is shown in Figure 3. Fibrinogen fouling on DOPA-pCB coated PDMS was limited to $10.4 \pm 3.0 \%$ of the uncoated PDMS surface. The effect of the DOPA-pCB coating and NO flux on platelet binding are shown in Figure 4. As a result of decrease protein adsorption, Platelet adsorption on DOPA-pCB coated PDMS fell to $23.3 \pm 7.4 \%$ of the uncoated control $(p<0.01)$. NO flux alone also reduced platelet binding significantly $(\mathrm{p}<0.05)$, albeit to a lesser degree $(79 \pm 5 \%$ of control). The combination of NO release and polycarboxybetaine coating further decreased platelet adsorption to $6.9 \pm 1.3 \%$ of that on untreated PDMS $(\mathrm{p}<0.001)$. This $93 \%$ reduction is 
close to the sum of the reduction in platelet adhesion caused by the pCB coating $(77 \%)$ and the NO (21\%) alone.

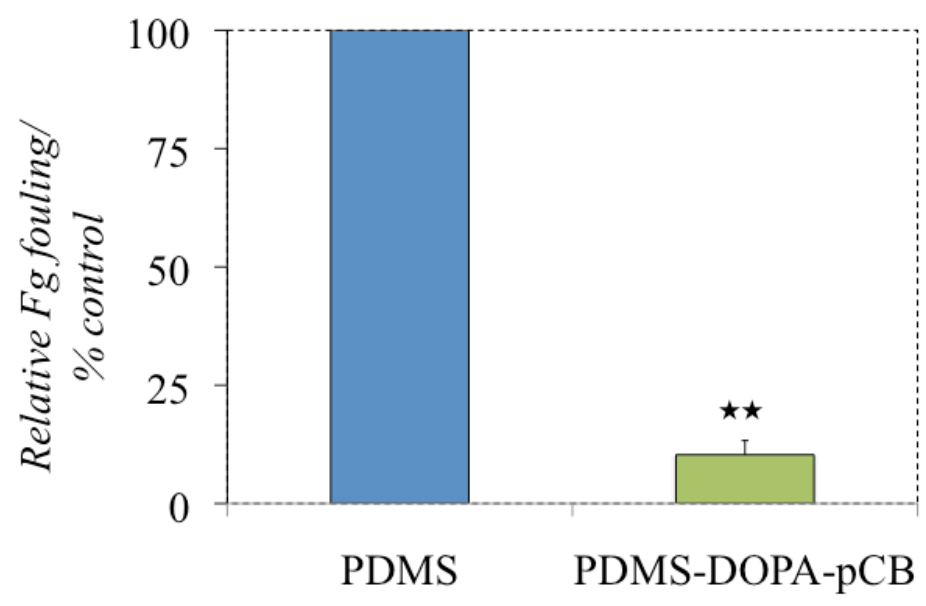

Substrate

Figure 3. (Fibrinogen fouling on uncoated and pCB-coated PDMS substrates. Data is expressed as percentage of fouling on uncoated substrates.)

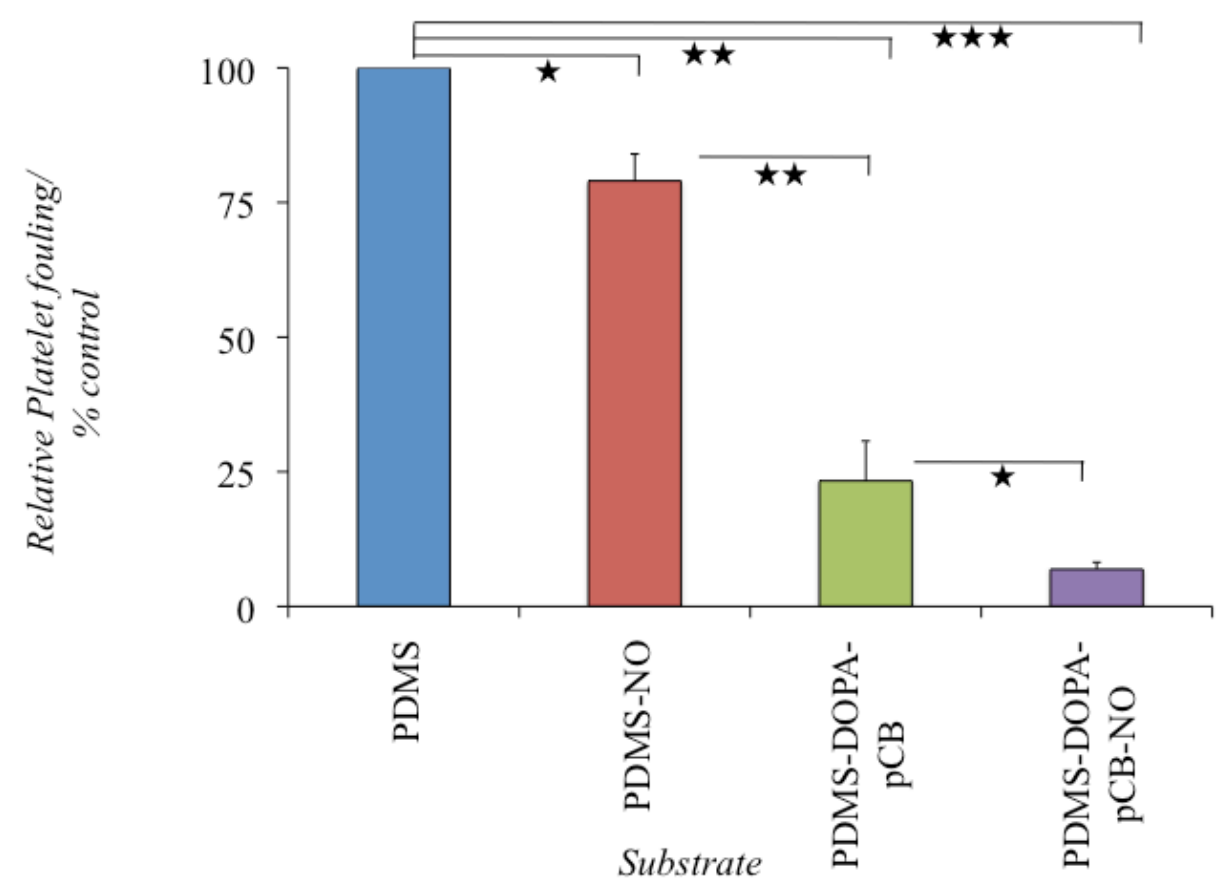

Figure 4. (Platelet adsorption on NO-releasing, DOPApCB-coated PDMS. The synergistic effect of coating and NO release on platelet adsorption is compared the effects of coating alone, NO release alone, and plain PDMS surface. Data is expressed as percentage of fouling on uncoated substrates.) 


\section{WILEY-VCH}

The quantities of surface bound platelets on substrates tested are shown in Table 2. Using the mean value of platelets that adsorbed onto unmodified PDMS as the baseline, surface nitric oxide release from PDMS was able to prevent over 2 million platelets from adsorbing. On the other hand, PDMS coated with pCB prevented over 8 million platelets from adsorbing. On PDMS substrates modified to release nitric oxide and with $\mathrm{pCB}$ coating, over 10 million platelets from adsorbing.

Table 2. (Platelet fouling on modified and unmodified polydimethylsiloxane substrates.)

\begin{tabular}{|l|l|l|}
\hline Substrate & Absorbance & $\begin{array}{l}\text { Platelet Fouling } \\
{\left[\times 10^{6} \text { cells* }{ }^{*-2}\right]}\end{array}$ \\
\hline PDMS & $6.26 \pm 2.70$ & $10.76 \pm 4.64$ \\
\hline PDMS-NO & $4.96 \pm 0.24$ & $8.52 \pm 0.41$ \\
\hline PDMS-pCB & $1.46 \pm 0.11$ & $2.50 \pm 0.18$ \\
\hline PDMS-pCB-NO & $0.45 \pm 0.01$ & $0.76 \pm 0.01$ \\
\hline
\end{tabular}

\subsection{Discussion}

Our findings confirm that the pairing of anti-platelet nitric oxide release and low fouling zwitterionic polycarboxybetaine polymer coating limits platelet fouling at a significantly higher level than achieved with either nitric oxide release or polycarboxybetaine alone.

Based on the known anti-clotting mechanisms of NO and hydrophilic pCB coating, we hypothesized that the effects of NO and pCB coating should be additive. In theory, this approach acts much like the endothelium by attacking two separate means of activating coagulation. The concept of incorporating several anticoagulant functions of the endothelium on an artificial surface is not new, but the systems being combined here have never been tested in tandem. The results support the additive hypothesis. DOPA-pCB coating alone reduced platelet adsorption to $23.3 \pm 7.4 \%$ of the uncoated control $(\mathrm{p}<0.01)$. The flux of 100 ppm NO alone also reduced platelet binding significantly $(\mathrm{p}<0.05)$, albeit to a lesser degree ( $79 \pm 5 \%$ of control). The combination of NO release and polycarboxybetaine coating further decreased platelet adsorption to $6.9 \pm 1.3 \%$ of that on untreated PDMS $(\mathrm{p}<0.001)$. This $93 \%$ 


\section{WILEY-VCH}

reduction is close to the sum of the reduction in platelet adhesion caused by the $\mathrm{pCB}$ coating (77\%) and the NO (21\%) alone.

Additive inhibition was expected because blood coagulation on artificial materials is initiated by clotting factors adsorbing and activating on the surface, but is accelerated by subsequent platelet activation via fibrinogen or thrombin. Here, $\mathrm{pCB}$ coating suppresses protein adsorption, reducing the amount of adsorbed fibrinogen, fibrin, and thrombin available to activate platelets. The NO blocks the propagation of any clot formation driven by platelets that were able to encounter these molecules by effecting conformational changes in platelets' thrombin and fibrinogen surface receptors.

These results compare favorably a similar study of this nature involving PMEA coated polypropylene and NO release. ${ }^{[17]}$ While PMEA coating reduced platelet adsorption to $82.6 \pm$ $9.2 \%$ of uncoated control, $\mathrm{pCB}$ coating studied here reduced platelet adsorption to $23.3 \pm$ $7.4 \%$ of uncoated. When PMEA coating was combined with 100 ppm NO flow, platelet adsorption was reduced to $30.3 \pm 6.8 \%$ of uncoated control. The combination of pCB coating with the same $100 \mathrm{ppm}$ NO flow (NO flux $=1.03 \pm 0.41 \times 10^{-10} \mathrm{~mol} / \mathrm{min} / \mathrm{cm}^{2}$ ) reduced platelet adsorption to $6.90 \pm 1.30 \%$ of uncoated control. With PMEA coating, it is estimated that it would require $200 \mathrm{ppm}$ of NO flow and an NO flux of $2.40 \pm 1.63 \times 10^{-10} \mathrm{~mol} / \mathrm{min} / \mathrm{cm}^{2}$ to achieve similar results. ${ }^{[17]}$ Thus, $\mathrm{pCB}$ allows for lower NO levels to be used without sacrificing biocompatibility, and lowers the risk of elevated methemoglobin concentrations (metHb). The NO flux $\left(1.03 \pm 0.41 \times 10^{-10} \mathrm{~mol} / \mathrm{min} / \mathrm{cm}^{2}\right)$ generated in this study is within the range of published endothelial NO flux levels. This level of NO flux is an order of magnitude lower than most papers showing effective inhibition of coagulation at an artificial surface. ${ }^{[2,8-}$ $11,17]$

Much like the effectiveness of NO depends on its flux, the effectiveness of the pCB coating depends on the its coverage of the base polymer (PDMS) and its stability. As the coating of hydrophobic materials (e.g. PDMS) with hydrophilic polymers (e.g. pCB) is a 


\section{WILEY-VCH}

challenge, further optimization methods are being investigated to develop best coating processes. ${ }^{[27]}$ They include i) dopamine assisted catechol chain end carboxybetaine methacrylate coating and ii) adding organic, water-miscible solvents into the coating solvent (TRIS buffer). The first method should increase hydrophobic interactions between the hydrophobic substrate and the dopamine assisted catechol chain end of carboxybetaine methacrylate. It has been reported that dopamine self-polymerize to polydopamine ${ }^{[28-31]}$ under basic conditions and this binds to the surface through adhesive interaction between catechol/surface and cohesive interaction among catechol. ${ }^{[32-38]}$ The exact mechanism for the formation of polydopamine is still not clear, although it was accepted that under basic conditions the catechol oxidizes to quinone, which then reacts with amine groups of dopamine. [29-31] While mixing a polymer with DOPA chain end (DOPA-pCB) along with dopamine, the DOPA end of the polymer chain would react to dopamine of polydopamine and thus becomes attached to polydopamine. This technique has been used to coat DOPA-pCB polymer on to a variety of different surfaces, including this study. Further improvements in coating efficiency could be achieved through the addition of water miscible solvents such as methanol and tetrahydrofuran. This should increase the wettability of hydrophobic surfaces to aid coating uniformity. These and other optimization methods are aimed at reducing fibrinogen fouling to within $\sim 5 \%$ of fouling on uncoated controls.

Despite these positive results, several questions must be answered to determine if this approach is beneficial in clinical applications. First, the stability of pCB coatings under longterm in vivo blood flow is unknown. ${ }^{[18]}$ The coating must remain attached and functional to maintain its anti-coagulant properties. Previous results demonstrate promise, however. Lightly cross-linked poly(carboxybetaine methacrylate) hydrogels were able to maintain normal glucose sensor function for 42 days in whole blood. ${ }^{[39]}$ Second, optimal and safe levels of NO are unknown. Larger NO concentrations will lead to more effective inhibition of platelets. A previous study by our group demonstrated complete elimination of platelet binding on bare 


\section{WILEY-VCH}

polypropylene using $500 \mathrm{ppm}$ of NO with a flux of $11.17 \times 10^{-10} \mathrm{~mol} \mathrm{~min} \mathrm{~cm}^{-1}$. However, larger NO flux could lead to elevated levels of metHb and impaired oxygen delivery in patients. The ideal safe level will depend on the surface area of the device. Smaller surface area devices, such as cannulae and catheters are unlikely to cause increase metHb at any reasonable NO flux, while larger surface area devices like artificial lungs have the potential to raise metHb levels at larger fluxes. For comparison, 10-80 ppm of inhaled NO has been used safely for days in patients, leading to significant increases in metHb of up to $5-7 \%$ but only in the 80 ppm groups. ${ }^{[40,41]}$ However, the adult lung has a huge surface area of $85 \mathrm{~m}^{2}$ or greater and should transfer markedly more NO than an adult artificial lung with a surface area of 1-2 $\mathrm{m}^{2}$. This suggests that $100 \mathrm{ppm}$ NO in an artificial lung should also be safe, but long-term in vivo testing must be done to verify.

The effect of our test surfaces on other cell types must also be worked out. Based on the physical properties of samples tested, there are three possible types of interactions that can take place between the sample surface and blood cells. Since the surfaces released NO gas, and presented $\mathrm{pCB}$ polymer brushes to flowing media, blood cells including platelets, red blood cells, and white cells would interact with pCB, NO, and PDMS to different degrees. NO can also bind to the heme centers of red blood cells, generating methemoglobin and temporarily reducing the oxygen-carrying capacity of blood. For this reason, methemoglobin levels are monitored when delivering NO to blood. Endogenous NO has been shown to have an effect on white cells. In our published extra-corporeal recirculation study, systemic white cell counts were significantly lower in the NO generating artificial lungs group than in control lungs, which did not generate NO. ${ }^{[9]}$ Other studies also accounted that increased endogenous NO levels is associated with decreased neutrophil sequestration. ${ }^{[44]}$ The nature of interaction between blood elements and pCB is different than with NO. Since surfaces coated with pCB groups are highly resistant to nonspecific adsorption due to electrostatically induced hydration, ${ }^{[38]}$ they may reduce inflammatory reactions from occurring as a result of higher levels of 


\section{WILEY-VCH}

activated complement and intrinsic coagulation cascade activation. The interaction between procoagulant PDMS and blood elements lead to protein adsorption, followed by platelet, red blood cell, white cell adhesion, and solid clot formation. While pCB is highly biocompatible with no known toxic effects, NO flux released from test samples is comparable to levels released by endothelial cells and thus will pose reversible effects on blood cells.

In addition the evaluation of resistance to clot formation by medical devices would greatly benefit from a standardized approach. There is, unfortunately, no established standard criterion for evaluating thrombogenicity in medical devices. Thus, strategies to evaluate and combat clot formation vary widely. Data on clot formation is based on a mixed bag of in vitro and in vivo test settings. The gold standard is long-term in vivo testing; however, there is no guarantee that these results will translate accurately to clinical use. Clot formation and device failure is based on a wide range of variables, many of which are never assayed, and remains highly variable from patient to patient and animal to animal. A standard translatable approach is certainly needed, but it is not yet known what is ideal.

The following limitations should also be considered in the interpretation of our results. First, both NO and surface-generated procoagulant molecules accumulate over time in our system because it is a closed in-vitro circuit. Such accumulation would not occur in-vivo due to NO sinks in the patient and clearance of procoagulant molecules. Second, NO flux will also be different in blood due to the presence of red blood cells. The red blood cells scavenge NO after it travels through the plasma exclusionary layer near the biomaterial surface. This sink will limit NO's effect in the bulk flow but also shrink the diffusional boundary layer and increase NO flux. Third, the effect of NO will vary slightly if NO is catalytically generated from donors in blood ${ }^{[7-9,23,25]}$ or supplied via the sweep gas in artificial lungs. In this later case, $\mathrm{NO}$ gas will react with $\mathrm{O}_{2}$ prior to delivery to the blood-contacting surface, reducing delivery of $\mathrm{NO}$ and increase delivery of nitrite and nitrate. 


\section{WILEY-VCH}

\section{Conclusion}

Both DOPA-pCB and NO flux significantly reduced platelet adhesion to biomaterial surfaces, with DOPA-pCB having a greater effect than NO. Together, they reduced platelet adhesion synergistically with a total platelet reduction that was similar to the sum of the reductions from each separately. The reduction in platelet adhesion with $\mathrm{pCB}$ was superior to that previously measured using a PMEA coating. Furthermore, the excellent resistance to fibrinogen fouling achieved using the pCB coating allowed for nearly complete (94\%) reduction in platelet adhesion with even low levels of NO flux $\left(1.03 \pm 0.41 \times 10^{-10} \mathrm{~mol} \mathrm{~min}^{-1}\right.$ $\mathrm{cm}^{-2}$ ). Thus, the pairing of low-level, anti-platelet NO release with DOPA-pCB has excellent promise toward surfaces with marked reductions in long-term coagulation.

\section{Experimental Section}

Surface attachment of zwitterionic polycarboxybetain polymer by "graft-to" method: Using already reported protocol ${ }^{[26,27,29,30,43]}$, Carboxybetaine methacrylate (CBMA) monomers were polymerized into DOPA-terminated pCB (DOPA-pCB) polymer via atomic transfer radical polymerization (ATRP). Dopamine is a highly adhesive molecule that essentially adheres and self-polymerizes at $\mathrm{pH}=8.5$ on many surfaces including PDMS. DOPA-pCB synthesis is shown in Figure 5A. First, dopamine (100mg) was conjugated to 2bromo isobutyl bromide $(\mathrm{Br}-\mathrm{i}-\mathrm{Bu}-\mathrm{Br})$ initiator $(1.5 \mathrm{ml})$ by first adding dopamine into a $50 \mathrm{ml}$ glass tube and covered with a rubber stopper. Then the stopper was wrapped with parafilm followed by purging the glass tube with nitrogen. While the glass tube was under vacuum, 20 $\mathrm{ml}$ of nitrogen-purged toluene (15 minutes) and $2 \mathrm{ml}$ of pyridine was added. The tube was then placed on ice and the initiator was added drop-by-drop over 30 minutes. The exothermic solution was left stirring overnight under nitrogen. The resulting DOPA-Br conjugate was vacuum dried for 2 hours after discarding its solvent. The conjugate was then lyophilized for 


\section{WILEY-VCH}

24hrs. In a fresh $50 \mathrm{ml}$ glass tube, $55 \mathrm{mg} / \mathrm{ml} \mathrm{CBMA} \mathrm{monomer} \mathrm{was} \mathrm{added} \mathrm{to} 20 \mathrm{ml}$ nitrogenpurged water $(1 \mathrm{hr})$ and covered with rubber stopper. The solution was then subjected to 5 cycles of 20 minutes of purging with nitrogen and 30 seconds vacuum and then left to stir for 15 minutes. $1 \mathrm{ml}$ of separately prepared complex copper solution was added to previous solution and left stirring overnight under nitrogen. The complex copper solution was prepared by adding $33 \mathrm{mg} \mathrm{CuBr}$ and $4 \mathrm{mg} \mathrm{CuBr}_{2}$ in a $10 \mathrm{ml}$ glass tube, followed by adding $2 \mathrm{ml}$ of purged water and $100 \mathrm{uL}$ of 1,1,4,7,10,10-Hexamethyltriethylenetetramine (HMTETA) and stirring for 10 minutes. The resulting polymer was deprotected in $10 \mathrm{ml}$ tetrahydrofuran (THF) with 5mg tetra-n-butylammonium fluoride (TBAF) to remove silanes on the DOPA of DOPA-pCB. The DOPA-pCB conjugate was vacuum dried for 2 hours after discarding its solvent and then lyophilized overnight.

DOPA-pCB polymer was then grafted onto PDMS substrates using 1:6 free DOPA to DOPApCB polymer dissolved in tris(hydroxymethyl)aminomethane (TRIS) buffer ( $\mathrm{pH}=8.5$ ). The grafting process is shown in Figure 5B. Substrates were first cleaned in methanol for 5 minutes and rinsed with water. They were then dried under nitrogen before immersing them in the above solution and initiating agitation for coating. 
A)

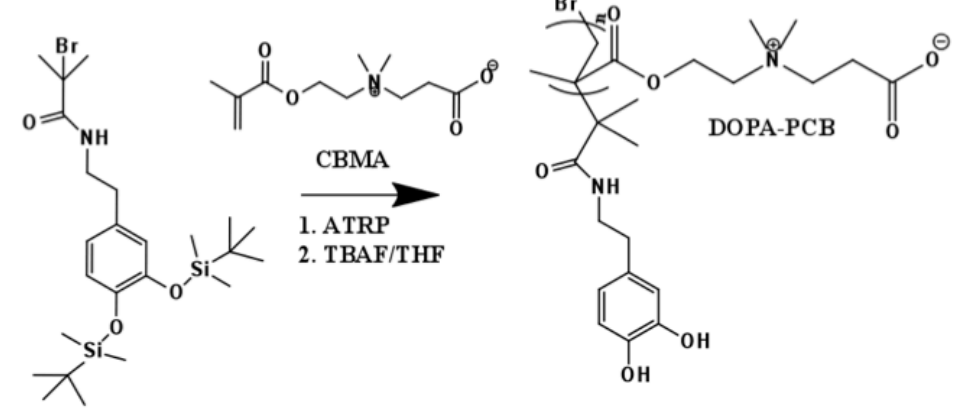

B)

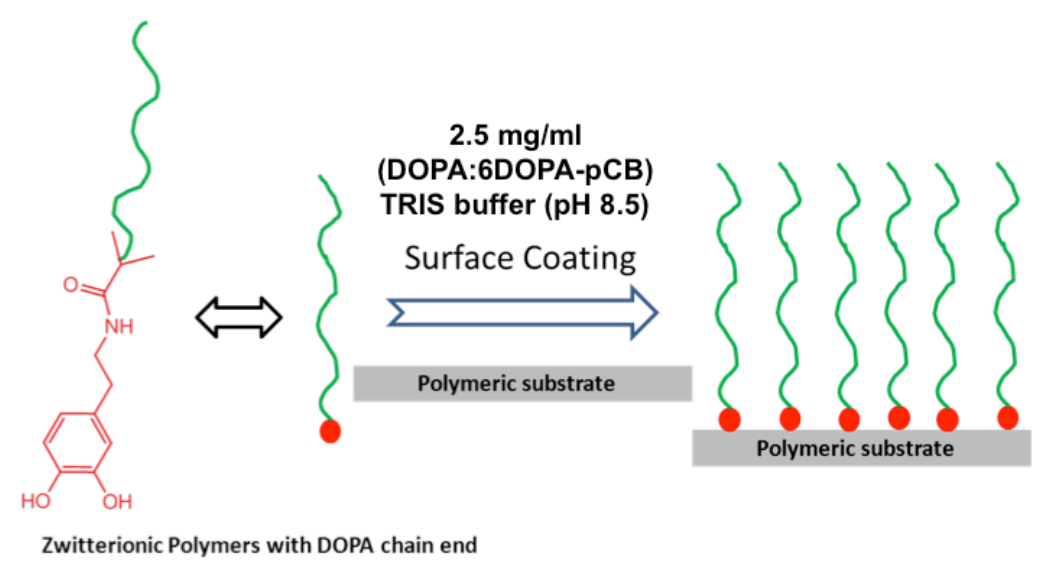

Figure 5. ((A) Dopamine-polyCarboxybetaine (DOPA-pCB) was synthesized via solution atomic transfer radical polymerization using an already reported protocol ${ }^{26}$. The reaction sequence starts with $A$ ) the reaction of dopamine (DOPA) linker with 2-bromo isobutyl bromide (Br-i-Bu-Br) initiator to form DOPA-Br, then 2) the reaction of DOPA-Br with polyCarboxybetaine methacrylate (CBMA) for form DOPA-pCB. B) Substrate's surface modification with DOPA-pCB polymer via pseudo one step "graft-to" coating approach. 1:6 free DOPA to DOPA-pCB were blended into a $2.5 \mathrm{mg} / \mathrm{ml}$ in tris(hydroxymethyl)aminomethane (TRIS) buffer $(\mathrm{pH}=8.5)$ and was used to bathe substrates for 6 hrs.))

DOPA served as the linker molecule between the substrate and DOPA-pCB polymer. As described in our published work ${ }^{[27]}$, the theory is that free catechol, DOPA either polymerizes into rafts in TRIS at $\mathrm{pH}=8.5$ and deposits onto PDMS or forms aggregates after deposition. The surface DOPA network then provides anchoring sites for the DOPA chain end of DOPApCB polymer brush via DOPA-DOPA polymerization. All solvents, chemicals, and complexes were purchased from Sigma Aldrich, St Louis MO.

After attachment, substrates were gently rinsed with deionized water to remove unbound or loosely attached DOPA-pCB and then stored in phosphate buffered saline, PBS (pH 7.34) under $4^{\circ} \mathrm{C}$ conditions for further testing. The samples stored in phosphate buffered 


\section{WILEY-VCH}

saline were then shipped on dry ice from University of Washington to the University of Michigan for biocompatibility testing. The modified surfaces were examined for $\mathrm{pCB}$ attachment using X-ray photoelectric spectroscopy. Detection of nitrogen indicated attachment of $\mathrm{pCB}$. Previous screening for optimal coating conditions using fouling data showed that 1 part DOPA to 6 parts DOPA-pCB suspended in TRIS buffer, resulting in a $2.5 \mathrm{mg} / \mathrm{ml}$ blend concentration, yields the lowest specific protein adsorption under specific coating conditions. ${ }^{[2]}$ These conditions include 1) using a $1.5 \mathrm{ml}$ of blend for every $4 \mathrm{~cm}^{2}$ of grafting surface area and 2) wet-grafting for $6 \mathrm{hr}$.

\section{Protein adsorption:}

A standard ELISA method was used to quantify protein adsorption binding to the surfaces using horseradish peroxidase-conjugated anti-IgG adsorption. ${ }^{[13]}$ The samples were first incubated for $1.5 \mathrm{~h}$ in human plasma fibrinogen dissolved in PBS (3.0 mg/ml, $\mathrm{pH} 7.34)$ in 24well plate. They were then rinsed $5 \mathrm{x}$ to wash out unbound fibrinogen and transferred into a new 24-well plate. 200 microliters of $1 \mu \mathrm{g} / \mathrm{mL}$ HRP-conjugated goat anti-human IgG antibody was added to each sample and incubated for $1 \mathrm{~h}$. This was followed by five rinses with PBS buffer. All samples (uncoated and the DOPA-PCB-100 coated) were moved to new wells. Next, $1 \mathrm{~mL}$ of $1 \mathrm{mg} / \mathrm{mL}$ o-phenylenediamine (OPD) in $0.1 \mathrm{M}$ citrate phosphate buffer (pH 5.5) containing $0.03 \%$ hydrogen peroxide was added. In the presence of hydrogen peroxide, peroxidase enzyme conjugated to anti-IgG catalyzes the oxidation of nearly colorless OPD to a soluble yellow-orange product. Peroxidase enzyme activity was stopped by adding $1 \mathrm{~mL}$ of $1 \mathrm{~N} \mathrm{HCl}$ after $15 \mathrm{~min}$. Finally, the absorbance of the yellow-orange color solution (intensity is proportional to the amount of protein adsorption) was then measured at $492 \mathrm{~nm}$. 


\section{WILEY-VCH}

The surface of modified PDMS was evaluated using X-ray photoelectron spectroscopy. The surface analysis experiments were done at Surface Analysis Recharge Center, NESAC/BIO supported by NIBIB grant EB-002027. All XPS spectra were taken on a Surface Science Instruments S-probe spectrometer. This instrument has a monochromatized Al Ka X-ray and a low energy electron flood gun for charge neutralization of non-conducting samples. The samples were fastened to the sample holder with double sided tape and run as insulators. Xray spot size for these acquisitions was approximately $800 \mu \mathrm{m}$. Pressure in the analytical chamber during spectral acquisition was less than $5 \times 10^{-9}$ Torr. Pass energy for survey spectra (to calculate composition) was $150 \mathrm{eV}$ and pass energy for high resolution scans was $50 \mathrm{eV}$. The take-off angle (the angle between the sample normal and the input axis of the energy analyzer) was $0^{\circ}\left(0^{\circ}\right.$ take-off angle $\sim 100 \AA$ sampling depth). The binding energy scales of the high-resolution spectra were calibrated by assigning the most intense C1s highresolution peak a binding energy of $285.0 \mathrm{eV}$. Two spots were analyzed on each sample.

\section{Bioreactor flow cell and test circuit:}

Custom-built, polycarbonate bioreactor flow cells were fabricated to facilitate simultaneous flows of complex media and NO. Test surfaces were inserted in the flow cell as shown in Figure 6, where blood plasma flows over the modified surface and NO travels from the gas phase, through the modified and unmodified surface, and then into the plasma. The bioreactor flow cell allows a total membrane surface area of $7.7 \mathrm{~cm}^{2}$ to be tested. Each flow cell was connected to a circuit consisting of 3/16" inner diameter Tygon tubes (Fisher Scientific, Pittsburg, PA) to form a closed serial circuit. After the circuits had been filled with plasma and all gas pockets eliminated, they were inserted into roller pumps (Stockert multiflow, Baxter Century Baxter Healthcare Cooperation Irvine, CA) to recirculate the plasma. 


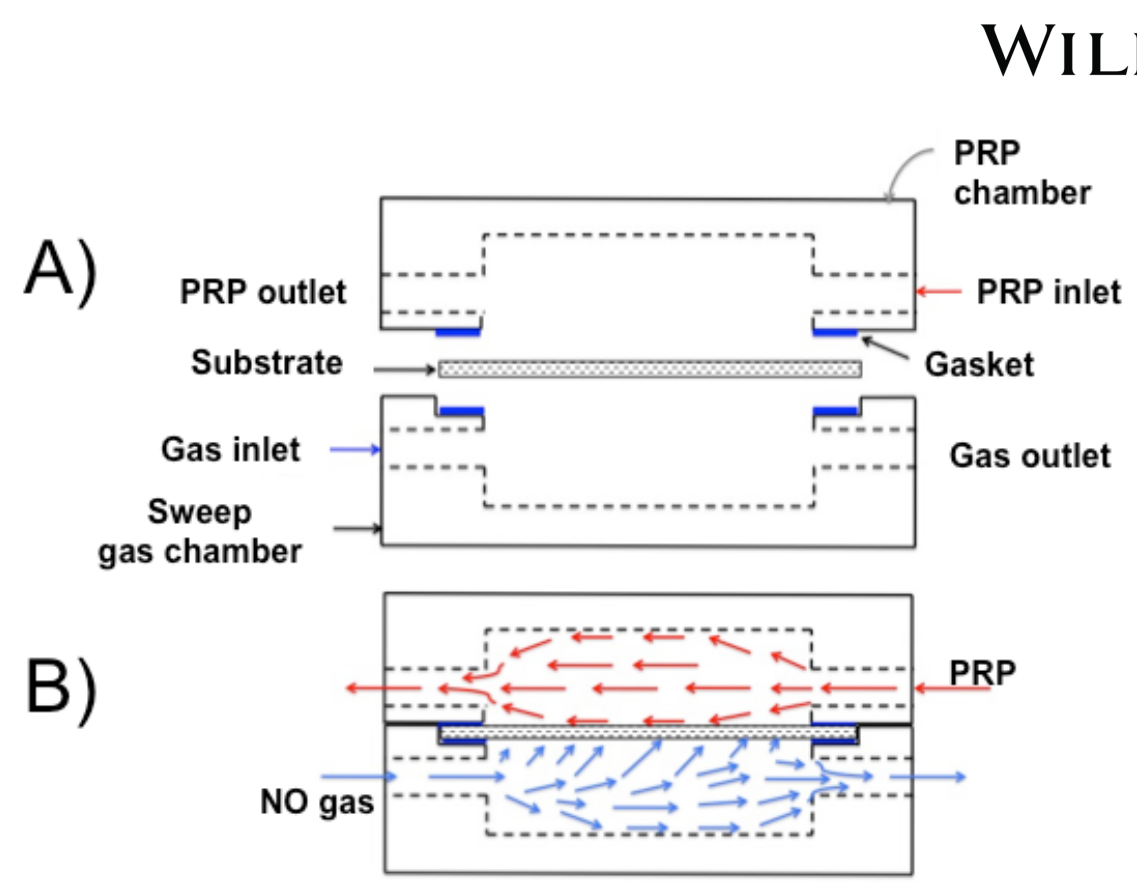

Figure 6. (Bioreactor flow cell; exploded view of flow cell showing platelet rich plasma (PRP) flow chamber, test membrane, and sweep gas flow chamber (A) and assembled bioreactor showing PRP and NO gas counter-current flow (B).)

\section{Quantifying nitric oxide flux from membranes:}

The methods for measuring NO flux from gas permeable membranes have been previously described. ${ }^{[17]}$ In brief, the relationship between sweep gas NO concentration and the membrane NO flux was determined using PBS recirculation within the bioreactor circuit. This is a conservative estimate of NO flux during our plasma experiments, below. In plasma, some NO would bind to proteins, leading to a slightly higher effective diffusivity. A total of $55 \mathrm{~mL}$ of PBS (25 $\mathrm{ml}$ to fill bioreactor $+30 \mathrm{ml}$ reservoir) was recirculated on one side of the membrane at $60 \mathrm{~mL} / \mathrm{min}$, while $\mathrm{N}_{2}$ with $100 \mathrm{ppm}$ and 500ppm of NO flowed over the opposite surface. Two NO concentrations were used here only to ascertain that membrane NO flux changes with NO concentration. PBS samples were taken before initiating flow and at $3 \mathrm{~min}$, $5 \mathrm{~min}$, 10min, $20 \mathrm{~min}$ and $30 \mathrm{~min}$ of recirculation. PBS NO concentrations were immediately measured with a Sievers Nitric Oxide Analyzer 280i (GE Instruments, Boulder, CO) using standard wet-phase measurement methods. The NO flux at each sampling time, $J_{N O}$ $\left(\mathrm{mol} / \mathrm{min} / \mathrm{cm}^{2}\right)$, was first calculated as $\left([\mathrm{NO}]_{\mathrm{c}}-[\mathrm{NO}]_{\mathrm{p}}\right) /\left(\left(\mathrm{t}_{\mathrm{c}}-\mathrm{t}_{\mathrm{p}}\right)^{*} \mathrm{~A}\right)$, where $[\mathrm{NO}]_{\mathrm{c}}$ and $[\mathrm{NO}]_{\mathrm{p}}$ are NO concentrations at the current and previous time points, $t_{c}-t_{p}$ is the sampling time 


\section{WILEY-VCH}

interval, and $\mathrm{A}$ is the membrane surface area. $J_{N O}$ at each time point was then used to calculate the NO mass transfer coefficient, $K(\mathrm{~cm} / \mathrm{min})$, for each time point. $K$ was calculated as $K=$ $J_{N O} /\left([\mathrm{NO}]_{\max }-[\mathrm{NO}]_{\text {measured }}\right)$, where $[\mathrm{NO}]_{\max }(\mathrm{mol} / \mathrm{ml})$ is the maximum achievable $[\mathrm{NO}]$ in PBS at the temperature and pressure from the NO gas concentration. Maximum fluid NO concentration, $[\mathrm{NO}]_{\max }$, was calculated as solubility(NO) $\mathrm{x} \mathrm{p}_{\mathrm{NO}}$, where solubility(NO) is the Henry's constant for solubility of NO in water at standard temperature and pressure (1.94 x $\left.10^{-3} \mathrm{~mol} \mathrm{~L}^{-1} \mathrm{~atm}^{-1}\right)^{[17]}$ and $\mathrm{p}_{\mathrm{NO}}$ is the partial pressure of $\mathrm{NO}$ in $\mathrm{N}_{2}$ gas obtained as $\mathrm{NO}(\mathrm{ppm}) \times 1 \mathrm{~atm}$.

\section{Platelet Adhesion Studies:}

The methods used here have been previously published. ${ }^{[17]}$ In brief, all platelet adhesion studies utilized freshly drawn sheep plasma. Animal handling and surgical procedures were approved by the University Committee on the Use and Care of Animals in accordance with University of Michigan and federal regulations. Sheep whole blood was gravity drawn into a blood transfer blood bag (1:10 acid citrate dextrose 4\% (ACD) anticoagulant, Sigma Aldrich, St Louis, MO). Whole blood from the bag was then carefully transferred to $50 \mathrm{~mL}$ centrifuge tubes and centrifuged at $730 \mathrm{rpm}$ for $20 \mathrm{~min}$ to generate PRP. Platelet rich plasma was then recovered, and the remaining blood was centrifuged at $2750 \mathrm{rpm}$ for $15 \mathrm{~min}$ to obtain platelet poor plasma (PPP). A Beckman-Coulter particle counter (Z1 Coulter Particle Counter, Indianapolis IN) was then used to determine platelet counts of the PRP and PPP. The PRP and PPP were then mixed to obtain $1 \times 10^{8}$ cells $/ \mathrm{mL}$.

Twenty-five $\mathrm{mL}$ of this mixture was injected into each bioreactor circuit. . Prior to injection, each bioreactor was fitted with a PDMS membrane that was uncoated or coated with pCB. The PRP flow rate was set to $60 \mathrm{ml} / \mathrm{min}$ as measured by a transonic flow probes (Transonic Systems, Ithaca, NY). Lastly, the sweep gas was nitrogen with $100 \mathrm{ppm}$ of NO set 


\section{WILEY-VCH}

at a flow rate twice the PRP flow rate. Based on modeling (see Results), this should lead to an approximately NO flux of $1.03 \pm 0.41 \times 10^{-10} \mathrm{~mol} \mathrm{~min}^{-1} \mathrm{~cm}^{-2}$.

To reduce biases created by run-to-run variability, four circuits were always run simultaneously such that four experimental conditions could be run with the same batch of plasma. In each run, four conditions were randomly chosen from all possible combinations of surfaces including PDMS, pCB-coated PDMS, PDMS with 100 ppm NO flow, and pCBcoated PDMS with 100 ppm NO flow. A total of N=5 runs were conducted for uncoated PDMS, PDMS coated with pCB, and PDMS with 100ppm NO and $\mathrm{N}=3$ for pCB-coated PDMS with 100 ppm NO flow. The membranes were then carefully removed from the bioreactors and rinsed $3 \mathrm{x}$ in PBS using a $3 \mathrm{~mL}$ pipette to wash away non-adhered blood plasma elements. The membrane was then sectioned lengthwise into two halves. One half was assayed for lactate dehydrogenase (LDH), and the other half was fixed for SEM analysis. Experimental runs with four circuits were repeated until five experiments were performed for each substrate coating and NO concentration combination.

A LDH assay kit (Cayman Chemicals Ann Arbor, 10008882) was used to quantify platelets adhered on the surface of each membrane. Each membrane section was incubated at $37^{\circ} \mathrm{C}$ in two $\mathrm{mL}$ of lysing reagent $(1 \%$ Triton-X100, $0.75 \%$ Bovine Albumin Serum in phosphate buffered saline) for $1 \mathrm{hr}$, with occasional agitation. The suspension was then assayed for LDH according to the kit manufacturer's protocol. To convert LDH concentration to the number of adhered cells, a calibration curve was first constructed. PRP $\left(7 \times 10^{8}\right.$ platelets $/ \mathrm{mL}$ ) was serially diluted with PPP to achieve platelet concentrations of $4.7 \times 10^{8}$, $2.4 \times 10^{8}, 1.2 \times 10^{8}, 6.0 \times 10^{7}, 3.0 \times 10^{7}, 1.5 \times 10^{7}$, and $7.5 \times 10^{6}$ cell $/ \mathrm{ml}$ and were assayed for LDH. A linear curve fit was then used to determine the relationship between absorbance from LDH and number of cells. This curve was found to be $\mathrm{LDH}_{\text {absorbance }}=0.119 \times$ platelets $\left(\times 10^{6}\right.$ cells $/ \mathrm{mL})+0.010,\left(\mathrm{R}^{2}=0.99\right)$ 


\section{WILEY-VCH}

To generate SEM images, the other half of the membrane was fixed with $2 \%$ gluteraldehyde (Sigma Aldrich, ST Louis MO) overnight and then dehydrated in a series of ethanol solutions and completely dried. The mid-section of the membrane was cut, sputtercoated with gold, and imaged by a Hitachi S-3200N scanning electron microscope $(15 \mathrm{kV})$.

\section{Data and Statistical Analysis:}

For fibrinogen adsorption, the absorbance of blank PBS solution at $492 \mathrm{~nm}$ was subtracted from the absorbance obtained from yellow-orange solutions in which samples were incubated to generate the absorbance due to fibrinogen. For quantitative comparison, all absorbance were presented as a percentage of the average fouling on uncoated PDMS samples (controls, $\mathrm{N}=5$ ). Similarly the raw LDH data were adjusted to remove background absorbance occurring on surfaces with zero platelet adherence. To do so, a control study was run using PBS only in the circuit, and these membranes were assayed for LDH in the described manner $(\mathrm{N}=4)$. The average background LDH data was then subtracted from the LDH concentrations measured in all studies. Adjusted LDH data from treated samples were then expressed as a percentage of the adjusted LDH concentration obtained from uncoated, control membranes after a normal PRP exposure study. Lastly, the calibration curve between LDH and platelet count was used to determine the number of adhered platelets, which was then divided by the surface area of test sample to give platelets $/ \mathrm{cm}^{2}$. One-way analysis of variance (ANOVA) with a Tukey's post hoc analysis was used to compare normalized intra-group fouling and LDH data using the SPSS (Chicago, IL). Statistical significance was set to $\mathrm{p}<0.05$. Numerical values are presented as mean $+/$ - standard deviation.

\section{Acknowledgements}

This work was supported by NIH grants R01 HL089043 and NIH 5T32HL007853-15. We thank Jiang lab at the University of Washington, Chemical Engineering department for 


\section{WILEY-VCH}

assisting with surface coatings. We also thank Dr. Robert Bartlett's Extracorporeal Life

Support Laboratory at the University of Michigan for their assistance.

\section{References}

[1] D.F. Larson, D. Arzouman, L. Kleinert, V. Patula, and S. Williams, Perfusion 2000, 15,13 .

[2] D.A. Palanzo, D.L. Zarro, N.J. Manley, R.M. Montesano, M. Quinn, B.A. Elmore, P.A. Gustafson, J.M. Castagna, Perfusion 2001,16, 279.

[3] N. Yoshinari, I. Fumito, I. Yoshiki, T. Katsuo, U. Shoichi, M. Shigeho, and Y. Shingo, Anesth. Analg. 1999, 89, 573.

[4] S. Jiang, and C. Zhiqiang, Advanced Materials 2010, 22, 920.

[5] M. Tanaka, T. Motomura, M. Kawada, T. Anzai, Y. Kasori, T. Shiroya, K. Shimura, M. Onishi, and A. Mochizuki, Biomaterials 2000, 21, 1471.

[6] U. Demirkilic, E. Kuralay, Y.H. Tatar, M. Kocakulak, C. Kocum, and H. Ayhan, Journal of Bioactive and compatible polymers 2002, 19, 395.

[7] K.A. Amoako, Archangelo C., T.C. Major, M.E. Meyerhoff, G. Annich, R.H. Bartlett, ASAIO journal 2012, 58, 238.

[8] K.A. Amoako and K.E. Cook, ASAIO journal 2011, 57, 539.

[9] K.A. Amoako, J.P. Montoya, T.C. Major, A.B. Suhaib, H. Handa, D.O. Brant, M.E., R.H. Bartlett, K.E. Cook, J. Biomed. Mater. Res. A. 2013, 10A, 3511.

[10] H. Zhang, G.M Annich, J. Miskulin, K. Osterholzer, S.I. Mertz, R.H. Bartlett, and M.E Meyerhoff, Biomaterials 2002, 23, 1485.

[11] T.C. Major, D.O Brant, C.P. Burney, K.A. Amoako, G.M. Annich, M.E. Meyerhoff, R.H. Bartlett, Biomaterials 2011, 32, 5957.

[12] E.J. Campbell, V. O’Byrne, P.W. Stratford, I. Quirk, T.A. Vick, M.C. Wiles, Y.P. Yianni, ASAIO J. 1994, 40, M853. 


\section{WILEY-VCH}

[13] D.M. Whelan, W.J. van der Giessen, S.C. Krabbendam, E.A. va Vilet, P.D. Verdouw, P.W. Serruys, H.M. van Beusekom, Heart 2000, 83, 338.

[14] R.M. Rapoport, M.B. Draznin, F. Murad, Nature 1983, 306, 174.

[15] R.M. Palmer, A.G. Ferrige, S. Moncada, Nature 1987, 337, 524.

[16] R. Howlett. Nature 1998, 395, 625.

[17] S. Gupta, K.A. Amoako, A. Suhaib, K.E. Cook, Advanced Material Interfaces 2014, 1(8), DOI: 10.1002/admi.201400012.

[18] W. Yang, H. Xue, L.R. Carr, J. Wang, S. Jiang, Biosensors and Bioelectronics, 2011, $26,2454$.

[19] Z. Zhang, H. Vaisocherova, G. Cheng, W. Yang, H. Xue, S. Jiang, Biomacromolecules 2008, 9.10, 2686.

[20] J. Ladd, Z. Zhang, S. Chen, J. C. Hower, S. Jiang, Biomacromolecules 2008, 9.5, 1357.

[21] H. Vaisocherova', W. Yang, Z. Zhang, Z. Cao, G. Cheng, M. Piliarik, J. Homola, and S. Jiang, Anal. Chem. 2008, 80.20, 7894.

[22] T.C. Major, D.O. Brant, M.M Reynolds, R.H. Bartlett, M.E. Meyerhoff, G.M. Annich, Biomaterials 2010, 31, 2736.

[23] K.A. Amoako, P.J. Montoya, T.C. Major, A.B. Suhaib, H. Handa, D.O. Brant, M.E. Meyerhoff, R. H. Bartlett, and K.E.Cook, Journal of Biomedical Materials Research Part A 2013, 101, 3511 .

[24] S.M. Lantvit, B.J. Barrett, M.M. Reynolds, J Biomed Mater Res Part A 2013, 101A, 3201.

[25] K.A. Amoako, Nitric oxide therapies for local inhibition of platelets' activation on blood-contacting surfaces. Diss., The University of Michigan, October 2011.

[26] Y. Zhu, H.S. Sundaram, S. Liu, L. Zhang, X. Xu, Q. Yu, J. Xu, S. Jiang. Biomacromolecules 2014, 15.5, 1845. 


\section{WILEY-VCH}

[27] H.S. Sundaram, X. Han, A.K. Nowinski, N.D. Brault, Y. Li, J.R. Ella-Menye, K.A. Amoako, K.E. Cook, P. Marek, K. Senecal, S. Jiang, Adv. Mater. Interfaces, 2014, 1.6, DOI: 10.1002/admi.201400071.

[28] S. Chen S. Jiang, Advanced Materials 2008, 20.2, 335.

[29] J.H. Waite, Nature Materials 2008, 7.1, 8.

[30] B.P. Lee, P.B. Messersmith, J.N. Israelachvili, J. Waite, Annual review of materials research 2011, 41, 99.

[31] H. Lee, S.M. Dellatore, W.M. Miller, B.P. Messersmith. Science 2007, 318.5849, 426.

[32] S.M. Kang, N.S. Hwang, J. Yeom, S.Y. Park, P.B. Messersmith, I.S. Choi, R. Langer, D.G. Anderson, H. Lee, Advanced functional materials 2012, 22.14, 2949.

[33] H. Lee H, N.F. Scherer, P.B. Messersmith. Proceedings of the National Academy of Sciences 2006, 103.35, 12999.

[34] T.H. Anderson, J. Yu, A. Estrada, M.U. Hammer, J.H. Waite, J.N. Israelachvili, Advanced functional materials 2010, 20.23, 4196.

[35] H. Zhao, J.H. Waite, Journal of Biological Chemistry 2006, 281.36, 26150.

[36] S.M. Kang, I. Yu, W.K. Cho, H.K. Shon, T.G. Lee, I.S. Choi, J.M. Karp, H. Lee, Angewandte Chemie International Edition 2010, 49, 9401.

[37] M.B. Gorbet, M.V. Sefton, Biomaterials 2004, 25, 5681.

[38] S. Jiang, C. Zhiqiang, Advanced Materials 2010, 22.9, 920.

[39] Y. Wei, B. Bai, L.R. Carr, A.J. Keefe, J. Xu, H. Xue, C. A. Irvin, S. Chen, J. Wang, S. Jiang, Biomaterials 2012, 33, 7945.

[40] D. Davidson, E.S. Barefield, J. Kattwinkel, G. Dudell, M. Damask, R. Straube, C.T. Chang, Pediatrics 1998, 10.3 (1998): 325.

[41] T.A. Nakagawa, A. Morris, R.J. Gomez, S.J. Johnston, P.T. Sharkey, A.L. Zaritsky, J Pediatr. 1998,131,63.

[42] C. Gao, G. Li, H. Xue, W. Yang, F. Zhang, S. Jiang, Biomaterials 2010, 31.7, 1486. 


\section{WILEY-VCH}

[43] G. Cheng, G. Li, H. Xue, S. Chen, J. Bryers, S. Jiang, Biomaterials 2009, 30, 5234.

[44] A. Roma and L.R. McGahren, J Pediatr Surg. 2004, 39, 48. 